\title{
Beam-driven plasma-based acceleration of electrons with density down-ramp injection at FLASHForward
}

\author{
J. Grebenyuk*, A. Martinez de la Ossa, T. Mehrling, J. Osterhoff \\ Deutsches Elektronen-Synchrotron DESY, Notkestr. 85, 22607 Hamburg, Germany
}

\section{A R T I C L E I N F O}

Available online 30 October 2013

Keywords:

Plasma-based acceleration

Beam-driven wakefield acceleration

Density down-ramp injection

\begin{abstract}
A B S T R A C T
We present simulations of injection of plasma electrons triggered by a negative gradient in the plasmadensity profile for beam-driven plasma-wakefield acceleration. Studies show the potential of this trapping technique for the production of narrow-energy bandwidth, femtosecond, high-current electron beams with a small transverse normalised emittance in the hundred nm range. Moreover, the accelerated beams feature strong longitudinal phase-space correlations with sub-percent uncorrelated energy spreads. Tuning of density down-ramp injection is explored by scanning the slope and length of the downward transition. It is found that the mechanism causes particle trapping over a wide experimentally accessible parameter range. All simulations were performed in three spatial dimensions with the particle-in-cell code OSIRIS [1]. The investigated driver-beam properties and the plasma profile resemble expected experimental conditions at the FLASHForward facility at DESY.
\end{abstract}

(c) 2013 Elsevier B.V. All rights reserved.

\section{Introduction}

Plasma-wakefield acceleration of electrons [2,3] is a rapidly developing novel-acceleration technology which allows to generate extreme electric-field gradients exceeding $10 \mathrm{GV} / \mathrm{m}$. In contrast to conventional radio-frequency accelerators, which are limited by breakdown of the acceleration structure to fields three orders of magnitude smaller, plasma-based techniques enable the creation of $\mathrm{GeV}$ beams on centimeter scales [4-6]. For this reason, plasma particle acceleration is regarded as a promising technology for compact and affordable accelerator-driven radiation sources such as free-electron lasers [7] and, ultimately, high-energy physics colliders $[8,9]$.

In plasmas, the accelerating cavity is created by a co-propagating wakefield driver, which can either consist of an ultra-short, intense laser pulse (laser-wakefield acceleration or LWFA [2]) or a high current-density, relativistic particle beam (plasma-wakefield acceleration or PWFA $[3,10]$ ). The quality of the beams produced from both plasma acceleration techniques has been making significant progress in recent years. In particular, LWFA has demonstrated the generation of high-quality, reproducible, and tunable electron beams in multiple setups [11-14]. Only recently have beam-driven acceleration experiments shown the ability to deliver narrow energy-bandwidth electron beams [15]. One of the most important aspects of facilitating such advances in PWFA, and hence for

\footnotetext{
* Corresponding author. Tel.: + 494089981825.

E-mail address: jgreb@mail.desy.de (J. Grebenyuk).
}

allowing this technology to be considered for future applications, is controlling and tuning the event of electron-beam injection into a plasma wake, a process which dictates main beam properties such as the minimum transverse emittance, energy spread, and peak current. There are two distinct possibilities for beam injection: trapping of an externally prepared, pre-accelerated and tailored particle beam, and injection of background plasma electrons. Understanding injection from an external source is important for staging of multiple plasma accelerators or for boosting the energy of beams from conventional accelerators in a plasma-based setup. External beam injection can be challenging to realize experimentally since it requires production of ultra-short particle beams, much shorter than the plasma wavelength, to maintain their quality [16], as well as accurate synchronization of the injector and the driver.

In this paper, we focus the discussion on the trapping of electrons directly from the plasma in a beam-driven acceleration scheme by means of density down-ramp injection (DDI) [17]. DDI is one of various proposals for the realization of internally controlled injection in PWFA, other methods are e.g. laser-induced ionization injection [18,19], wakefield-induced ionization injection [20], and magnetically triggered injection [21]. Of these techniques only DDI has been successfully demonstrated in LWFA [14,22,23] and is of particular interest for future implementation in PWFA owing to its low complexity and tunability. We study density down-ramp injection by means of three-dimensional particle-in-cell (PIC) simulations with driver beam parameters corresponding to expected experimental conditions at the FLASHForward facility at DESY. These studies show the potential of the DDI technique for the production of narrow-energy bandwidth, femtosecond, high-current electron 
beams with a small transverse normalized emittance in the hundred nm range.

\section{Density down-ramp injection}

Density down-ramps in the propagation direction of a plasma wake (longitudinal direction) facilitate electron trapping from the background plasma into the accelerating and focusing phase of the wakefield. The mechanism allows for control over the injection process and the occupied phase by controlling the plasma-density profile. This enables tailoring of beam parameters, i.e. the energy spread, charge, current, and emittance.

In case of a plasma-density profile $n(z)$ which is changing along the wake-propagation direction $z$, the plasma-oscillation wavenumber $k_{p}$ also depends on $z$ :

$k_{p}(z)=\sqrt{\frac{4 \pi e^{2}}{m_{e} c^{2}}} \sqrt{n(z)}$

where $m_{e}$ is the electron mass, $e$ is the electron charge and $c$ is the speed of light. Therefore, the wake phase has an additional $z$ dependence $\psi=k_{p}(z)(z-c t) t$ denoting time. Thus, assuming a fixed wake phase with $d \psi / d t=0$, the wake phase velocity $v_{p}$ can be calculated according to Ref. [25]

$v_{p}(z)=c /\left(1+\frac{\zeta}{k_{p}(z)} \frac{d k_{p}}{d z}\right)$.

Here, $\zeta=z-c t$ is a co-moving coordinate. Taking into account Eq. (1), the change in wave number along $z$ can be written as $d k_{p} / d z=\left(k_{p} / 2 n\right) d n / d z$. For $d n / d z<0$, i.e. a negative plasmadensity gradient, $v_{p}$ decreases and the wake slows down. This effect relaxes the condition for longitudinal wave-breaking, i.e. reduces the velocity which plasma electrons have to achieve to be trapped, and hence, enables particle injection [17,24].

Simultaneously, in the presence of the longitudinal density gradient, plasma electrons experience a phase shift. The plasma wavelength $\lambda_{p}$ is inversely proportional to $\sqrt{n}$, causing an increase of $\lambda_{p}$ in the density downslope. The resulting phase shift can be expressed. The resulting phase shift can be expressed [25] as

$\Delta \psi=\psi_{0} \sqrt{1-\frac{n_{1}}{n_{0}}}$

with $\psi_{0}$ being the initial electron phase and $n_{0}$ and $n_{1}$ the initial and final plasma density of the ramp, respectively.

Two different regimes for DDI have been proposed which depend on the length of the density transition $L_{\text {ramp }}$. The original method [17] suggests injection from a gentle plasma-density gradient $k_{p} L_{\text {ramp }}>1$, where wave breaking and trapping of electrons happen due to a slow-down of the plasma-wave phase velocity. Using an ultra-short ramp [26] with $k_{p} L_{\text {ramp }} \leq 1$ facilitates injection of electrons with small phase spread due to a sudden rephasing into the accelerating and focusing phase. The latter regime is challenging to realize experimentally. In the following, our studies will focus on the long-ramp regime.

\section{FLASHForward at DESY}

FLASHForward is a novel-acceleration-techniques research beamline at the FLASH facility at DESY. It will include a set of experiments to advance the field of beam-driven wakefield acceleration in plasmas, among them exploration of DDI. The simulations presented in this paper have been performed in order to prepare the FLASHForward experimental setup and thus should be verified when the experiments commence in 2016. The following beam parameters were assumed for the simulations: Gaussian beam profiles of rms length $\sigma_{z}=8.4 \mu \mathrm{m}$ and rms width $\sigma_{x, y}=5 \mu \mathrm{m}$, a total charge of $180 \mathrm{pC}$ corresponding to a peak current of $2.5 \mathrm{kA}$, an energy of $1 \mathrm{GeV}$ with a relative energy spread of $1 \%$, and a normalized transverse emittances of $\varepsilon_{x}=\varepsilon_{y}=1 \mu \mathrm{m}$.

\section{Simulation setup and results}

The plasma-density profile used in the simulations is depicted in Fig. 1. An initial increase from vacuum to a density of $n_{0}=10 \times$ $10^{17} \mathrm{~cm}^{-3}$ is followed by a $100 \mu \mathrm{m}$ plateau of constant density and a linear decrease to density $n_{1}=1 \times 10^{17} \mathrm{~cm}^{-3}$ over a length of $L_{\text {ramp }}=60 \mu \mathrm{m}$. Following this down-ramp for triggering injection, a plateau of constant density $n_{1}$ allows for particle acceleration to high energies. $n_{1}=10^{17} \mathrm{~cm}^{-3}$ corresponds to a plasma skin-depth of $k_{p}^{-1}=16.8 \mu \mathrm{m}$ and wavelength of $\lambda_{p}=106 \mu \mathrm{m}$. The numerical investigation was conducted in a simulation frame with a moving window of box dimensions $20 \times 15 \times 15 k_{p}^{-3}$ with a cell size of $0.04 k_{p}^{-1}$ in each dimension. Eight particles per cell were used for both, driver and plasma.

Fig. 2 shows plasma and driver charge densities after $5.4 \mathrm{~mm}$ of propagation distance. An electron bunch is injected into the first wake period owing to the integrated density down-ramp. The injection process is illustrated in Fig. 3, which shows the longitudinal phase space $\left[z, p_{z}\right]$ (where $p_{z}$ is the longitudinal momentum) of plasma electrons: first wave-breaking occurs due to a decrease of wake phase velocity (top plot), and then electrons are trapped in the focusing and accelerating phase of the wake (bottom plot).

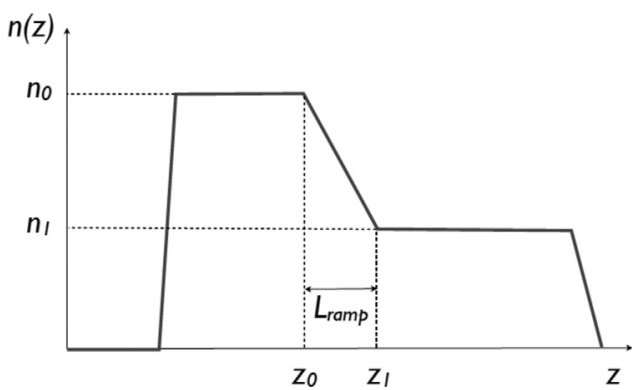

Fig. 1. Schematic representation of the plasma-density profile as a function of the longitudinal coordinate $n(z)$. An electron bunch is injected on the down-ramp transition between $z_{0}$ and $z_{1}$. Subsequently, its energy is boosted at constant density $n_{1}$ with $z \geq z_{1}$

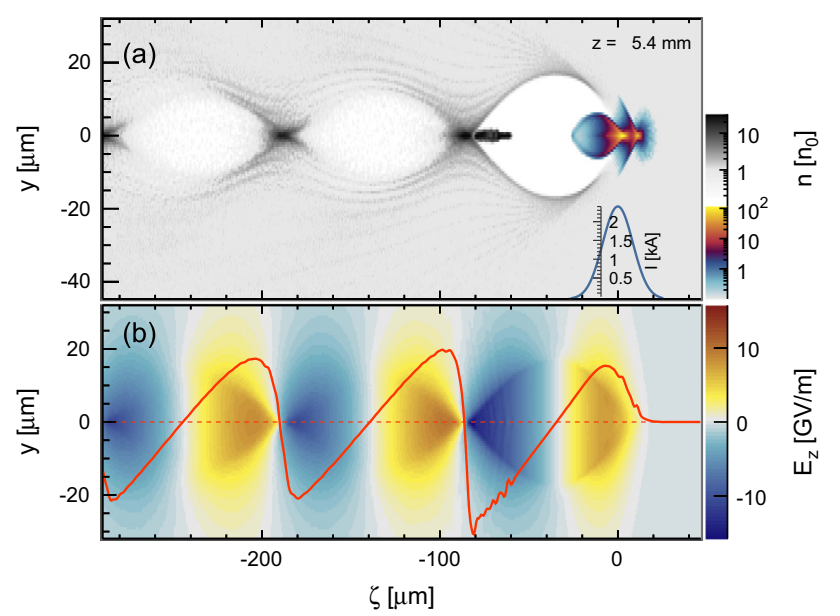

Fig. 2. (a) Charge density of the plasma and driver beam. (b) The longitudinal electric fields after $5.4 \mathrm{~mm}$ of propagation distance. An electron bunch was injected in the first plasma period at a density down-ramp transition. 

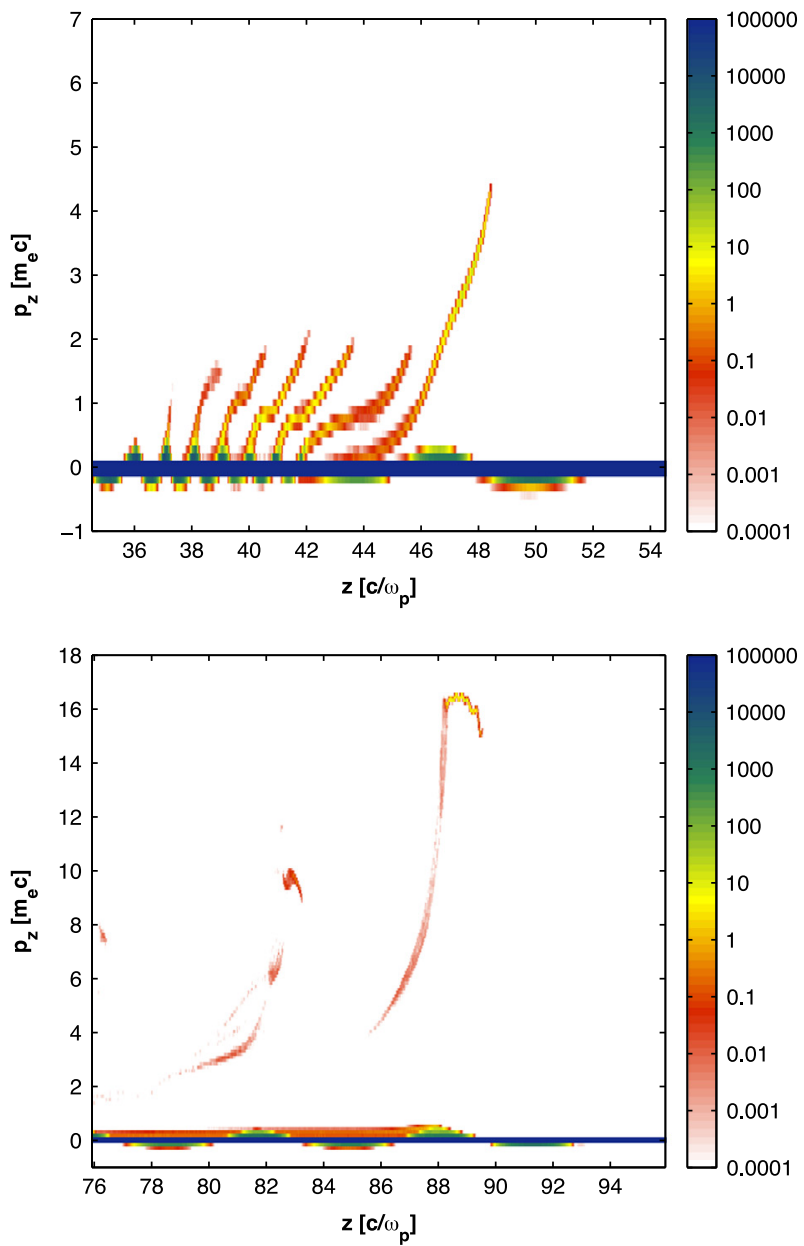

Fig. 3. Phase space $\left[z, p_{z}\right]$ on the density down-ramp during wave-breaking (top) and after particle trapping (bottom).

After the density ramp the injected bunch is further accelerated at constant density with field gradients of $10 \mathrm{GV} / \mathrm{m}$ until depletion of the driver beam occurs. After $14.5 \mathrm{~cm}$ of propagation through plasma the witness bunch has gained $1.5 \mathrm{GeV}$. Its projected relative energy spread is $11 \%$ originating from the phase spread at injection time, the rms beam sizes are $\sigma_{x}=0.5 \mu \mathrm{m}, \sigma_{y}=0.45 \mu \mathrm{m}$, and $\sigma_{z}=5.1 \mu \mathrm{m}$ with a total charge of $32 \mathrm{pC}$. The projected normalized transverse emittances are $\varepsilon_{x}=0.58 \mu \mathrm{m}$ and $\varepsilon_{y}=0.40 \mu \mathrm{m}$. An analysis of the process shows that the normalized emittance is small initially after trapping, $\varepsilon_{x}=\varepsilon_{y}=0.06 \mu \mathrm{m}$, but then grows rapidly caused by betatron-phase mixing until it saturates [16]. The average beam current is $I=484 \mathrm{~A}$.

The beam energy spectrum, longitudinal phase-space properties, and sliced emittance and current are depicted in Fig. 4. The longitudinal phase space $\left[z, p_{z}\right]$ is strongly correlated owing to a strongly varying accelerating field strength along the length of the beam. Therefore, the uncorrelated energy spread is below $0.5 \%$ while the absolute energy spread exceeds $10 \%$. Moreover, the sliced normalized transverse emittance is found to be below $0.2 \mu \mathrm{m}$ with a sliced current exhibiting an almost flat-top profile of $0.7 \mathrm{kA}$. These values correspond to the peak beam brightness which exceeds $0.35 \times 10^{17} \mathrm{~A} \mathrm{~m}^{-2} \mathrm{rad}^{-2}$ and is up to one order of magnitude higher than used in modern free-electron lasers (FELs) $[27,28]$. Since FEL performance is dependent on beam brightness, controlled injection on a density ramp may provide beams with the potential to drive the next-generation compact, highbrightness FEL source. The non-linearities in $\left[z, p_{z}\right]$ phase space can be attributed to beam-loading effects, and may be omitted by
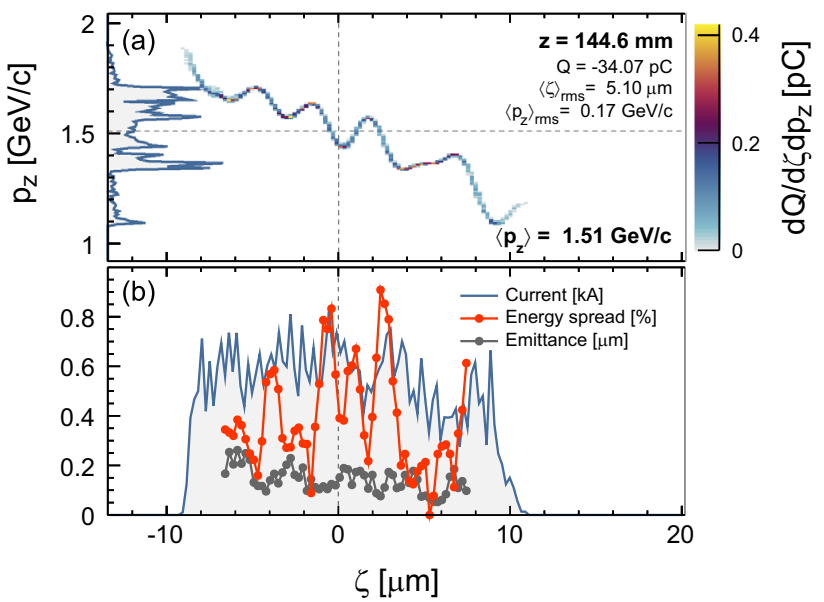

Fig. 4. (a) Energy spectrum and longitudinal phase-space $\left[z, p_{z}\right]$, and (b) sliced energy spread, normalized transverse emittance and peak current of the injected beam after $14.5 \mathrm{~cm}$ of propagation in plasma.

Table 1

Injected beam properties after $20 \mathrm{~mm}$ of propagation distance for different downramp lengths. The results are obtained from 3D OSIRIS simulations. The beam properties are found to depend weakly on the ramp length.

\begin{tabular}{lccc}
\hline Ramp length $(\mu \mathrm{m})$ & 60 & 200 & 500 \\
\hline Charge $(\mathrm{pC})$ & 32 & 35 & 37 \\
Mean energy $(\mathrm{MeV})$ & 216 & 212 & 203 \\
RMS length $\sigma_{z}(\mu \mathrm{m})$ & 5.2 & 5.5 & 5.7 \\
Radial RMS $(\mu \mathrm{m})$ & 0.57 & 0.82 & 0.78 \\
Total length $(\mu \mathrm{m})$ & 20 & 21 & 24 \\
Norm. trans. emittance $(\mu \mathrm{m})$ & 0.15 & 0.22 & 0.18 \\
Average current $(\mathrm{kA})$ & 0.5 & 0.5 & 0.5 \\
Peak current $(\mathrm{kA})$ & 0.9 & 0.9 & 0.9 \\
\hline
\end{tabular}

injecting beams with less charge on less steep gradients $n_{1} / n_{0}$ (see Section 5).

\section{Density ramp parameter scans}

Two sets of 3D parameter scans were performed to estimate the tunability of the DDI-induced beam as well as its sensitivity to experimental uncertainties arising from fluctuations in the initial conditions. Parameters of the driver beam are set to be the same for all cases. The first scan includes simulations with different values for $L_{\text {ramp }}$, while the slope of the ramp $s=L_{\text {ramp }} /\left(n_{1}-n_{0}\right)$ is kept constant. The value of $n_{1} / n_{0}$, i.e. the step in density at the ramp, is set to larger values for longer ramps. The injected beam properties, i.e. its charge, mean energy, length, transverse size (radial rms $\sigma_{r}=\sqrt{\sigma_{x}^{2}+\sigma_{y}^{2}}$ ), transverse normalized emittances, and the current, after $20 \mathrm{~mm}$ of propagation distance are presented in Table 1. Three values of $L_{\text {ramp }}$ are considered: $60 \mu \mathrm{m}, 200 \mu \mathrm{m}$, and $500 \mu \mathrm{m}$, which correspond to ramp lengths in terms of plasma wavelength of $0.57 \lambda_{p}, 1.87 \lambda_{p}, 4.7 \lambda_{p}$. Simulations show that for all values of $L_{\text {ramp }}$ the beam is injected, and its quality remains similar in terms of normalized transverse emittance, bunch size and peak current. Injection on a longer ramp results in slightly higher charge injected, which leads to slower acceleration. For all cases longitudinal phase space is strongly correlated, and sliced quantities exhibit similar properties as presented in Fig. 4. The absolute energy spread remains close to constant for all cases, on the order of $10 \%$. Our results show that the injected beam properties weakly depend on the length of the ramp as long as the slope is kept 
Table 2

Injected beam properties for different plasma density steps, i.e. down-ramp slopes. The results are obtained from 3D OSIRIS simulations. The beam properties were found to depend strongly on the ramp slope.

\begin{tabular}{lccc}
\hline Density step $n_{1} / n_{0}$ & 10 & 3 & 1.2 \\
\hline Charge $(\mathrm{pC})$ & 32 & 15 & 2 \\
Mean energy $(\mathrm{MeV})$ & 216 & 273 & 320 \\
RMS length $\sigma_{z}(\mu \mathrm{m})$ & 5.2 & 2.8 & 0.6 \\
Radial RMS $(\mu \mathrm{m})$ & 0.56 & 1.08 & 0.92 \\
Total length $(\mu \mathrm{m})$ & 20 & 13 & 3 \\
Norm. trans. emittance $(\mu \mathrm{m})$ & 0.15 & 0.26 & 0.4 \\
Average current $(\mathrm{kA})$ & 0.5 & 0.5 & 0.5 \\
Peak current $(\mathrm{kA})$ & 0.9 & 0.7 & 0.7 \\
\hline
\end{tabular}

constant, and longer ramps, which allow for more straightforward experimental setups than short ramps, induce similar-quality DDI beams.

A second scan includes 3D PIC simulations with constant $L_{\text {ramp }}=60 \mu \mathrm{m}$, while the slope of the ramp is varied by changing the step in density $n_{0} / n_{1}$. Three of these density steps are studied: 10,3 , and 1.2 . The results after $20 \mathrm{~mm}$ propagation distance are presented in Table 2. For all simulated plasma steps DDI-induced beams are observed. Simulations show that the slope of the ramp has a large influence on the injected beam. Less steep profiles induce smaller charge and shorter bunches with larger transverse normalized emittances. This imposes limits on the level of control necessary for the stability of plasma-density profiles in DDI experiments.

\section{Conclusions}

Simulations show that injection on a density down-ramp in a beam-driven wakefield accelerator at the FLASHForward beamline will allow for production of ultra-short, high-current, smallemittance beams. The predicted slice properties raise hopes for a potential application of these beams to drive compact, highbrightness light sources such as FELs. Parameter scans show that the injected beam quantities are weakly dependent on the ramp length and mainly affected by the slope of the density transition. This makes DDI an attractive injection mechanism for a future implementation in PWFA owing to its low complexity and tunability.

\section{Acknowledgments}

We would like to thank the OSIRIS team (IST/UCLA) for providing access to the OSIRIS code, with special thanks to $\mathrm{J}$. Vieira and R. Fonseca. We acknowledge the grant of computing time by the Jülich Supercomputing Centre on JUQUEEN under Project no. HHH09. We would like to thank DESY IT for their support concerning simulations and data storage at DESY and we acknowledge the Alexander von Humbolt Foundation for financial support.

\section{References}

[1] R.A. Fonseca, et al., in: Lecture Notes in Computer Science, vol. 2331, 2002, p. 342;

R.A. Fonseca, et al., Plasma Phys. Control. Fusion 50 (2008) 124034.

[2] T. Tajima, J.M. Dawson, Phys. Rev. Lett. 43 (1979) 267.

[3] V. Veksler, in: Proceedings of CERN Symposium on High Energy Accelerators and Pion Physics, vol. 1, 1956, p. 80

[4] W.P. Leemans, et al., Nat. Phys. 2 (2006) 696.

[5] I. Blumenfeld, et al., Nature 445 (2007) 741.

[6] X. Wang, et al., Nat. Comm. 4 (2013) 1988.

[7] F. Grüner, et al., Appl. Phys. B 86 (2007) 431.

[8] W. Leemans, E. Esarey, Phys. Today 62 (3) (2009) 44.

[9] C.B. Schroeder, et al., Phys. Rev. ST Accel. Beams 13 (2010) 101301.

[10] P. Chen, et al., Phys. Rev. Lett. 54 (1985) 693.

[11] J. Faure, et al., Nature 444 (2006) 737.

[12] J. Osterhoff, et al., Phys. Rev. Lett. 101 (2008) 085002.

[13] N.A.M. Hafz, et al., Nat. Photon. 2 (2008) 571.

[14] A.J. Gonsalves, et al., Nat. Phys. 7 (2011) 862.

[15] V. Yakimenko, Plenary talk at 1st European Advanced Accelerator Concepts Workshop 2013, 2013.

[16] T. Mehrling, et al., Phys. Rev. ST Accel. Beams 15 (2012) 111303.

[17] S. Bulanov, et al., Phys. Rev. E 58 (1998) R5257.

[18] B. Hidding, et al., Phys. Rev. Lett. 108 (2012) 035001.

[19] F. Li, et al., Phys. Rev. Lett. 111 (2013) 015003.

[20] A. Martinez de la Ossa, et al., in: Proceedings of the 1st European Advanced Accelerator Concepts Workshop 2013, 2013.

[21] J. Vieira, et al., Phys. Rev. Lett. 106 (2011) 225001.

[22] C.G.R. Geddes, et al., Phys. Rev. Lett. 100 (2008) 215004.

[23] A. Buck, et al., Phys. Rev. Lett. 110 (2013) 185006.

[24] A. Brantov, et al., Phys. Plasmas 15 (2008) 073111

[25] G. Fubiani, et al., Phys. Rev. E 73 (2006) 026402.

[26] H. Suk, et al., Phys. Rev. Lett. 86 (2001) 1011.

[27] S. Schreiber, et al., in: Proceedings of the 2010 International Particle Accelerator Conference, TUPEO04, 2010. 〈http://www.JACOW.org $\rangle$.

[28] J.N. Galayda, et al., J. Opt. Soc. Am. B 27 (11) (2010) B106. 